Supporting information

\title{
Resonance Raman Determination of Chromophore Structures of Heliorhodopsin Photointermediates
}

\author{
Taito Urui, ${ }^{+}$Misao Mizuno, ${ }^{+}$Akihiro Otomo, ${ }^{+\S}$ Hideki Kandori, ${ }^{\ddagger}$ and Yasuhisa Mizutani ${ }^{+*}$ \\ 'Department of Chemistry, Graduate School of Science, Osaka University, 1-1 \\ Machikaneyama, Toyonaka, Osaka 560-0043, Japan

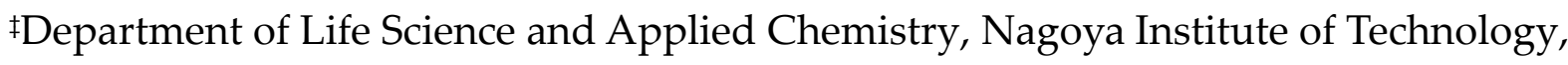 \\ Showa-ku, Nagoya, Aichi 466-8555, Japan.
}

\$Present address: Institute for Molecular Science, 5-1 Higashiyama, Okazaki 444-8787, Japan.

*Corresponding author: E-mail: mztn@chem.sci.osaka-u.ac.jp 

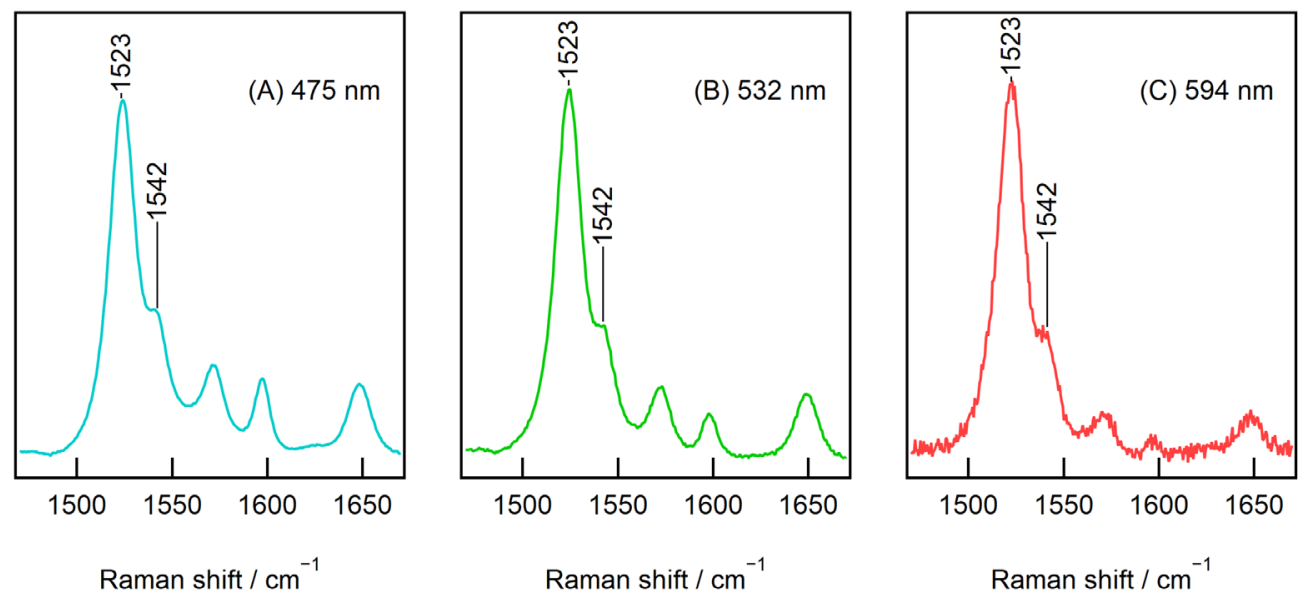

Figure S1. Probe wavelength dependence of the $v(C=C)$ bands of HeR 48C12. Panels A, B, and C show spectra measured with the probe light of 475,532 , and $594 \mathrm{~nm}$. The intensity ratios of the of the $v(C=C)$ doublet were almost constant for the three spectra. Absorption maximum wavelengths are different for the retinal chromophore with different structures. Thus, the retinal chromophore with different structures exhibits a different Raman excitation profile. If the $v(C=C)$ doublet is due to the presence of two species, the intensity ratios of the $v(C=C)$ doublet changes when the wavelength of the probe light is changed. However, the intensity ratios of the $v(C=C)$ doublet were almost constant for the probe wavelength of 475,532 , and $594 \mathrm{~nm}$. Invariance of the intensity ratio at the probe wavelength wider than $100 \mathrm{~nm}$ demonstrated that the $v(C=C)$ doublet is due to the single species. 

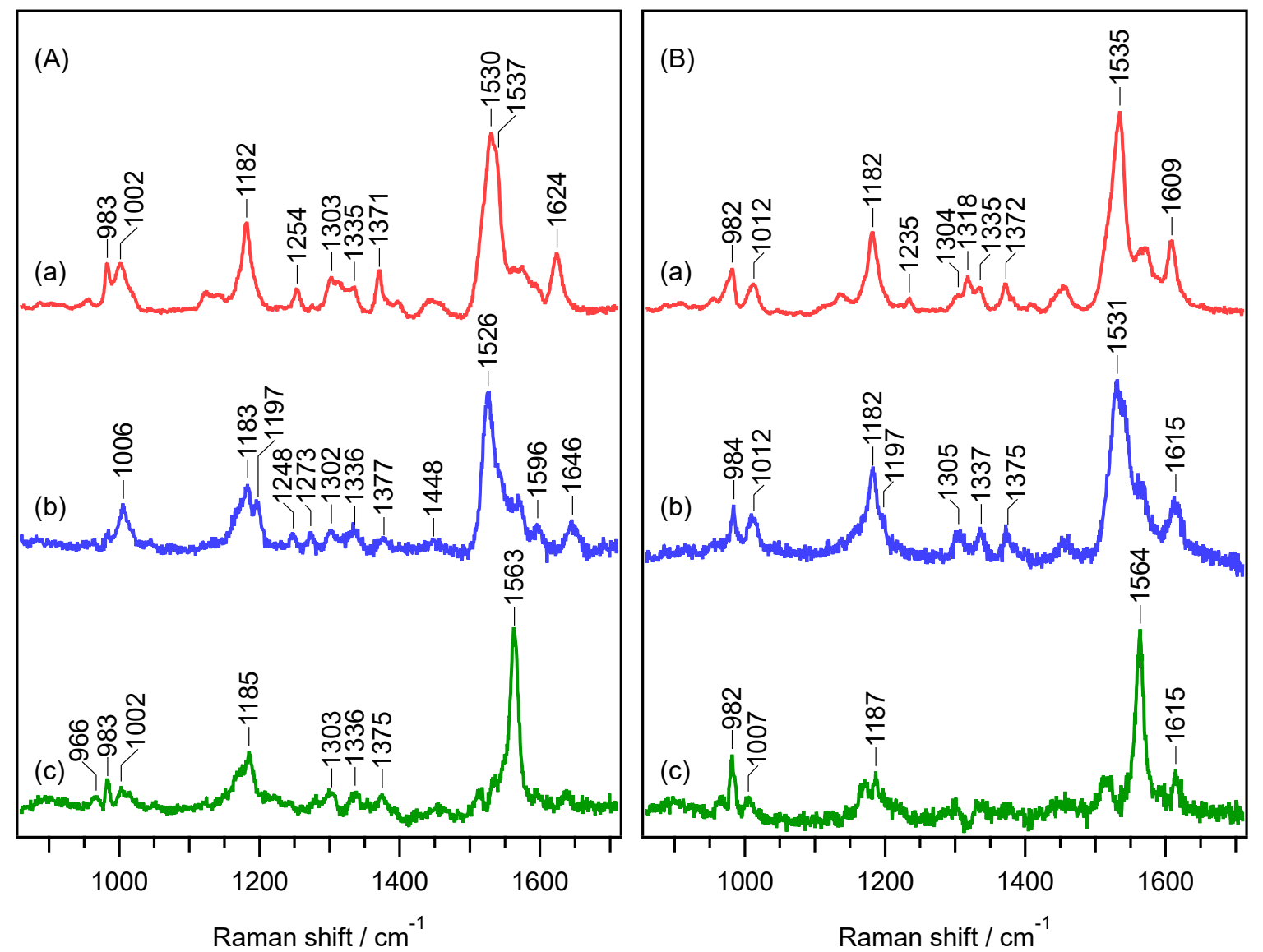

Figure S2. Decomposed resonance Raman spectra from traces $\mathrm{d}-\mathrm{f}$ of Figure 1 by calculating the linear combinations of the three traces. Traces $\mathrm{a}-\mathrm{c}$ are attributed to the $\mathrm{L}_{1}, \mathrm{~L}_{2}$, and $\mathrm{M}$ intermediates, respectively. Panel $A$ and $B$ show the spectra for the samples in $\mathrm{H}_{2} \mathrm{O}$ and $\mathrm{D}_{2} \mathrm{O}$, respectively. 


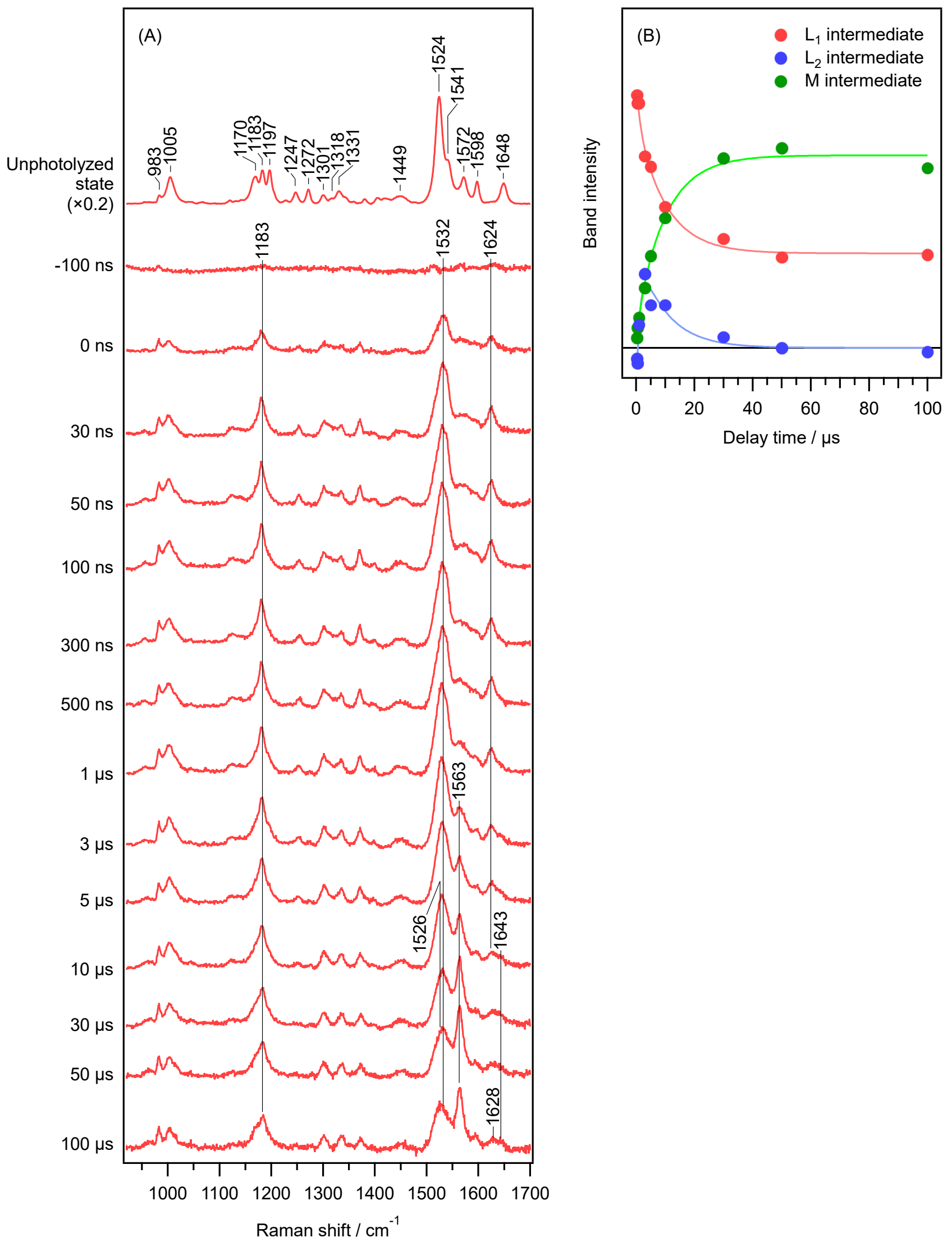


Figure S3. Time-resolved resonance Raman data of $\mathrm{HeR} 48 \mathrm{C} 12$ in $\mathrm{H}_{2} \mathrm{O}$ buffer at more delay times than in Figure 1. (A) Time-resolved spectra in in the nanosecond to microsecond range. The pump and probe wavelengths were 532 and $475 \mathrm{~nm}$, respectively. The time resolution of the measurements was $\sim 50 \mathrm{~ns}$. The spectrum of the buffer and the emission background were subtracted. The top traces are the Raman spectrum without the pump beam and thus are the spectra of the unphotolyzed state. The other spectra are time-resolved difference spectra obtained by subtracting the spectral contribution of the unphotolyzed state from the Raman spectrum obtained with the pump beam at each delay time. (B) Temporal intensity changes of the $v(\mathrm{C}=\mathrm{C})$ bands attributed to $\mathrm{L}_{1}$ (red), $\mathrm{L}_{2}$ (blue), and $\mathrm{M}$ (green) intermediates. While the $\mathrm{L}_{1}$ intermediate is diminant in submicrosecond range, the $\mathrm{M}$ intermediate is formed through the $\mathrm{L}_{2}$ intermediate on the microsecond timescale. Solid lines are best fits. For the fitting, populations of the $\mathrm{L}_{1}, \mathrm{~L}_{2}$, and $\mathrm{M}$ intermediates were calculated for the following scheme. ${ }^{1}$

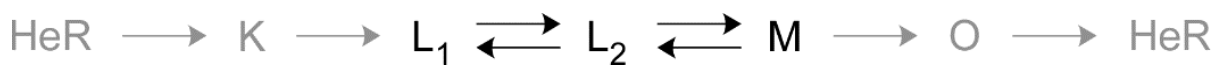




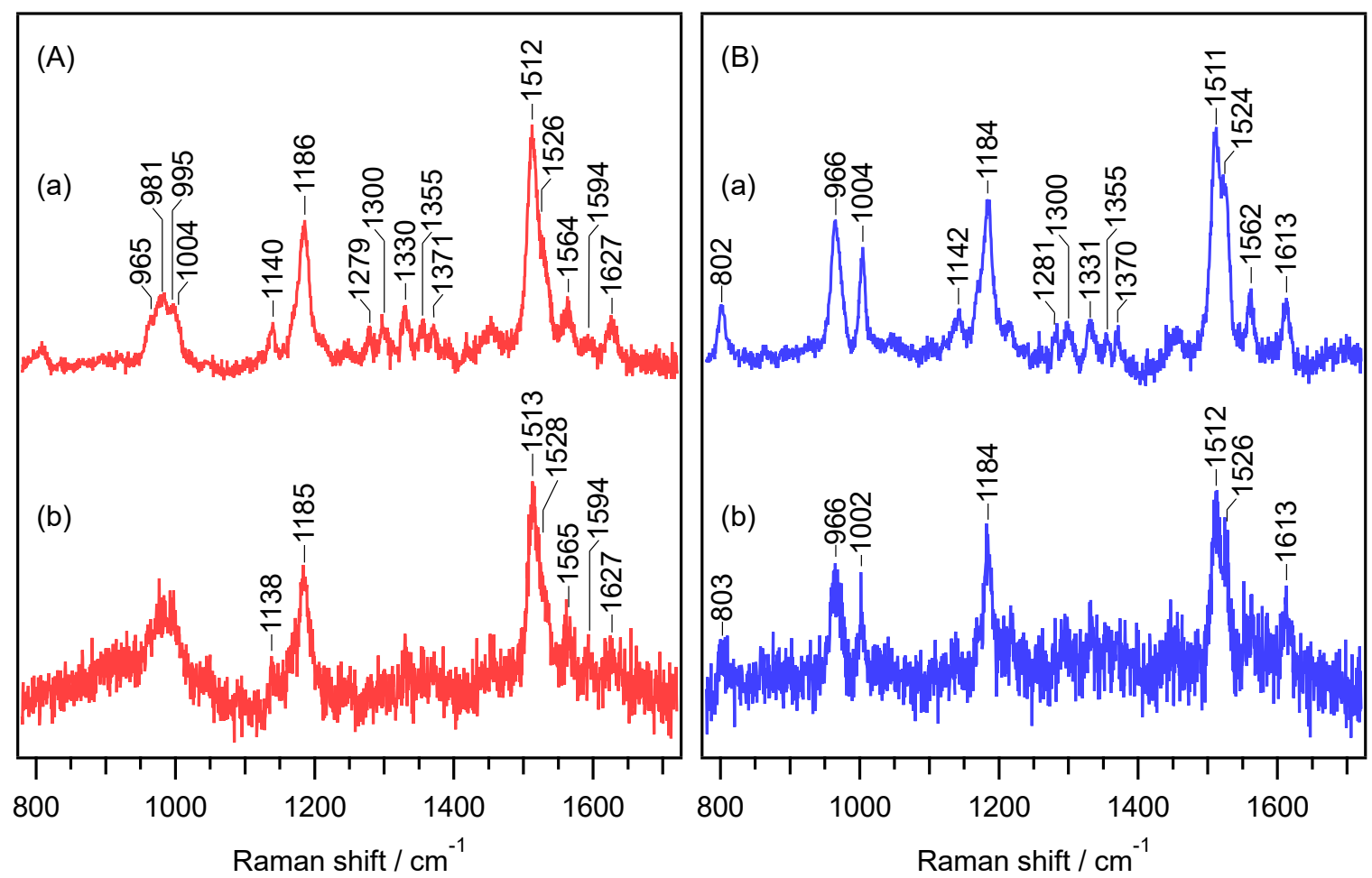

Figure S4. Resonance Raman spectra of the $\mathrm{O}$ intermediate of $\mathrm{HeR} 48 \mathrm{C} 12$ probed at $594 \mathrm{~nm}$ in $\mathrm{H}_{2} \mathrm{O}(\mathrm{A})$ and $\mathrm{D}_{2} \mathrm{O}$ (B) buffers. (a) Time-resolved spectra of the $\mathrm{O}$ intermediate after 532-nm photoexcitation (delay time, 2 $\mathrm{ms}$ ). (b) Spectra of the $\mathrm{O}$ intermediate in a photostationary state under the continuous illumination by green light centered at $530 \mathrm{~nm}$ emitted by LED (Thorlabs, M530L2-C1). The spectra of the unphotolyzed state, the buffer, and the emission background were subtracted. 


\section{References}

1. Johnson, K. A., [61] Rapid Kinetic Analysis of Mechanochemical Adenosinetriphosphatases. In Methods Enzymol., Academic Press: 1986; Vol. 134, pp 677-705. 